\title{
Radiographic imaging for Ilizarov limb lengthening in children
}

\author{
C.E.Blane ${ }^{1}$, J.E. Herzenberg ${ }^{2}$ and M. A. DiPietro ${ }^{1}$ \\ Departments of ${ }^{1}$ Radiology, Section of Pediatric Radiology, and ${ }^{2}$ Surgery, Division of Orthopedic Surgery, \\ University of Michigan Hospitals, Ann Arbor, Michigan, USA
}

Received: 22 January 1990; accepted: 10 July 1990

\begin{abstract}
The Ilizarov method for limb lengthening is rapidly gaining popularity in North America. Use of this new technique has necessitated modifications in radiographic protocol. Initial imaging problems gained from our experience with twenty children are detailed including accurate centering for the plain films, correcting for magnification to accurately measure the distraction gap and the expected radiographic appearance of the regenerate bone. Ultrasonography has potential value in accurately measuring the distraction gap and in imaging the new bone prior to radiographic appearance. Since overly fast distraction inhibits bone formation and overly slow distraction leads to premature consolidation, ultrasound may serve a useful role in the qualitative evaluation of new bone formation in Ilizarov limb lengthening, enabling the orthopedic surgeon to tailor the distraction rate to the particular child.
\end{abstract}

The Ilizarov apparatus and method have recently been introduced in North America and are rapidly gaining popularity as the method of choice for lengthening acquired and congenitally short limbs. The technique was developed in the Soviet Union over the past 30 years by Gavriil A. Ilizarov. The Ilizarov apparatus consists of a circular external fixator frame which maintains the position of the long bone by tensioned thin transfixion wires (Fig. 1 a). Distraction and compression are achieved by threaded rods connected to the circular external frame. Angular deformities are corrected by hinge mechanisms. Ilizarov devised a percutaneous osteotomy ("corticotomy") where care is taken to leave the periosteum intact and avoid damage to the medullary canal [1-5]. After an initial latency period of 5-15 days, distraction is begun at a rate of $0.25 \mathrm{~mm}$ four times daily. Once the desired lengthening has been achieved, the frame is left in place until the regenerate bone is sufficiently ossified to support the limb $[5]$.
Imaging of the distraction gap is required to measure the lengthening and to document new bone formation. Distraction can be slowed or advanced in proportion to the new bone formation. Overly fast distraction will inhibit new bone formation while overly slow distraction will lead to premature consolidation. The child's age, underlying bony pathology, and anatomic location of the corticotomy affect the potential rate of new bone formation. The rate of distraction must be adjusted according to the radiographic appearance of new bone in the distraction gap.

Plain film documentation of the new bone formation is the current standard utilized. It is usually possible to identify the new bone as linear streaks appearing between 3 and 9 weeks after distraction is initiated [6]. However, the surgeon must often continue distraction for several weeks without visual documentation of new bone formation. This can be worrisome.

The distraction gap can be measured accurately on plain film, provided the radiographic beam is centered to the distraction site and magnification is taken into consideration. The actual bulk of the apparatus lifts the limb 10-15 centimeters away from the film. Our early experience at the University of Michigan with Ilizarov limb lengthening in children has drawn our attention to many problems in radiographic imaging and interpretation. In addition, we have studied the potential usefulness of sonography to assess the developing new bone and accurately determine the corticotomy gap length [6-8].

\section{Materials and methods}

Twenty children aged 4-19 years (mean 12.6 years) underwent Ilizarov limb lengthening. A total of 26 corticotomies (distraction gaps) were created, and five children were lengthened simultaneously in two anatomic locations. The underlying etiologies of limb length discrepancy were post-traumatic growth arrest in eight children, multiple enchondromatosis in two children, congenital pseudarthrosis of the tibia in three patients (two with neurofibromatosis), bi- 


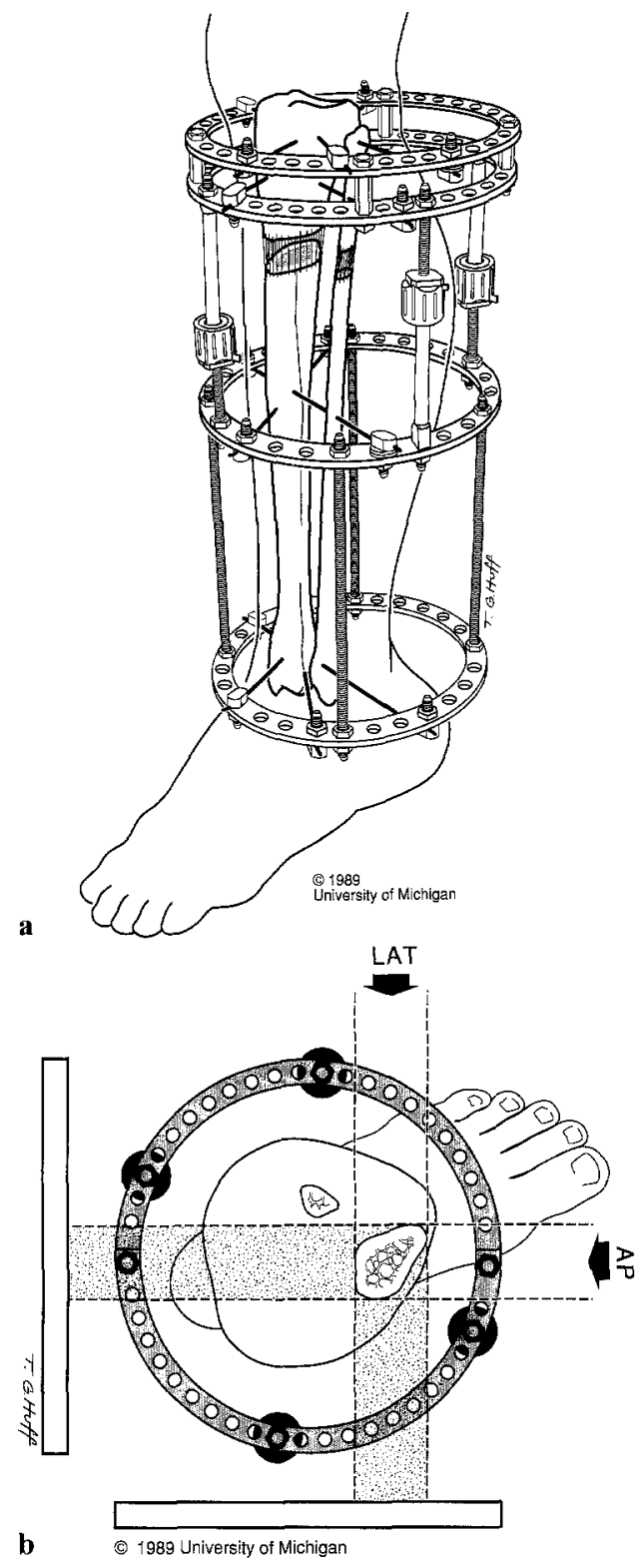

Fig. 1a, b. a The line diagram demonstrates the apparatus in position. There are 2 rings proximal and 2 rings distal to the corticotomy. Plain threaded rods are shown between rings \#3 and \#4. Graduated telescopic rods are illustrated between rings \#2 and \#3. b The cross-sectional view shows the orientation of these connecting posts or rods

lateral Blount's disease in one patient, unilateral Blount's in another, congenitally short tibia in three patients, proximal femoral focal deficiency in one patient and one patient had multiple congenital anomalies with shortening and contractures. Real-time ultrasound utilizing a $5 \mathrm{MHz}$ transducer in linear array was performed at various times during the lengthening and compared to the plain radiographs.

\section{Results and observations}

During the early phases of distraction, it is important to center the radiographic beam to the corticotomy site, in order to "gun site" down the distraction gap. If the beam is not centered to the distraction gap it may be impossible to discern if the gap is indeed separating. Failure of a gap to separate after one-two weeks of distraction implies premature consolidation or an incomplete corticotomy. While the patients can sometimes indicate the location of the distraction gap, the orthopedic clinicians have found it useful to include a line drawing on the radiographic consultation request form illustrating the exact location of the corticotomy in relation to the external fixator rings. Thus the request for a radiograph of the tibia in Figure 1 a might ask the technologist to center the beam $2-3 \mathrm{~cm}$ distal and parallel to the second ring from the top.

Additional care should be taken to ensure the vertical connecting rods (either long threaded rods, or graduated telescopic distractors) are aligned to avoid superimposition on the corticotomy gap (Fig $1 b$ ). The technologist may slightly rotate the limb so as to allow the beam to pass through the distraction gap without interference from the vertical distraction rods. This is particularly true when the surgeon uses the graduated telescopic rods shown between rings \#2 and \#3 in Figure 1a, because they are bulkier than the plain threaded rods shown between rings \# 3 and \# 4 in Figure 1 a.

Calculating the magnitude of the distraction gap is important confirmation for the clinician that the patient is following the precise instructions governing the distraction rate. Towards the end of the distraction it is important to know the exact distance of the gap length in order to achieve the preoperative goal of length gain. Scanograms can be unreliable due to magnification problems from the bulky apparatus and the occasional inability to straighten a joint. A radiopaque ruler can be fixed by Velcro bands to the limb at the level of the long bone for the radiograph. A commercially available triangular marker with three radiopaque points precisely $4 \mathrm{~cm}$ apart can also be placed on the limb for a standard (Fig. 2). Comparison of plain film measurement of the distraction gap, utilizing some method to correct for magnification, and ultrasound measurement indicates that accurate measurement of the distraction gap can be made when there is equal distraction on all sides. Asymmetric distraction is used to correct angular deformities. In unequal distraction the ultrasound measurements must be made in at least two locations corresponding to the maximum and minimum distraction gap sites.

During the first few weeks of distraction, no bone is seen on the radiographs (Fig. 3a). Linear streaks of calcification indicate early new bone formation on the plain films which typically appears near the cut ends of the bone (Fig. 3b). A similar linear streaky appearance of increased echogenicity is noted on the ultrasound (Fig. $3 \mathrm{c}, \mathrm{d}, \mathrm{e}$ ). The new bone formation could be identified on ultrasound by $2-3$ weeks postoperatively (1-2 weeks after initiation of distraction) despite a variable appearance on plain radiographs from 2 weeks in two patients, 3 weeks in seven patients, 4 weeks in three patients, 5 weeks in one patient to 6 weeks in another three patients. In patients with ultrasound studies prior to the radiographic appearance of the new bone formation, sonography revealed the linear streaks of new bone on the sonogram at least one week, and in two cases three weeks, prior to the plain film documentation (Fig.3). In no patient did the plain film appearance of the new bone formation preceed the ultrasound documentation, though ten patients did not have 

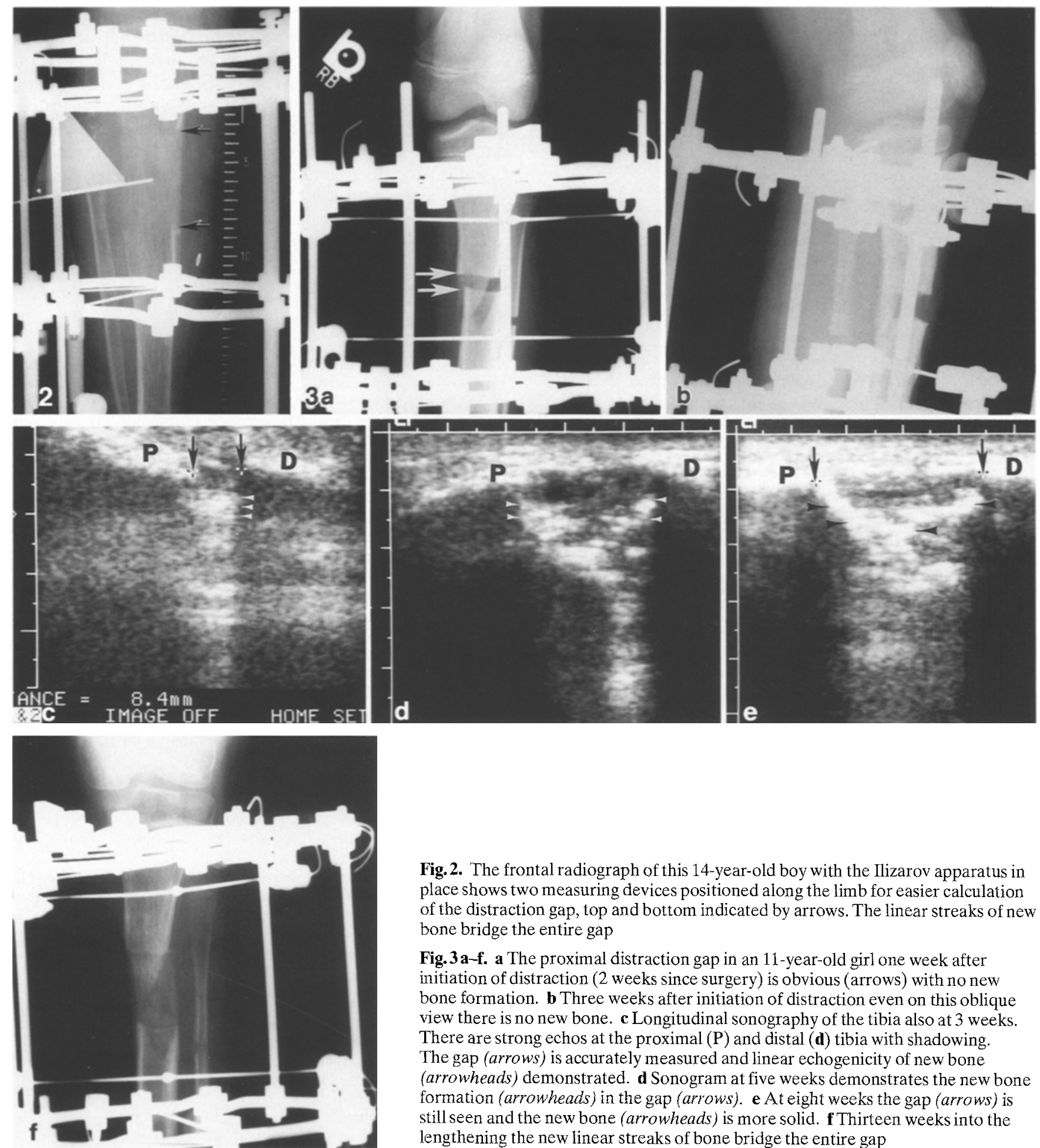

Fig. 2. The frontal radiograph of this 14-year-old boy with the Mizarov apparatus in place shows two measuring devices positioned along the limb for easier calculation of the distraction gap, top and bottom indicated by arrows. The linear streaks of new bone bridge the entire gap

Fig. 3a-f. a The proximal distraction gap in an 11-year-old girl one week after initiation of distraction ( 2 weeks since surgery) is obvious (arrows) with no new bone formation. b Three weeks after initiation of distraction even on this oblique view there is no new bone. c Longitudinal sonography of the tibia also at 3 weeks There are strong echos at the proximal (P) and distal (d) tibia with shadowing. The gap (arrows) is accurately measured and linear echogenicity of new bone (arrowheads) demonstrated. d Sonogram at five weeks demonstrates the new bone formation (arrowheads) in the gap (arrows). e At eight weeks the gap (arrows) is still seen and the new bone (arrowheads) is more solid. $\mathbf{f}$ Thirteen weeks into the lengthening the new linear streaks of bone bridge the entire gap

sonography until later in their course and five patients did not have ultrasound studies. Ultrasound of the corticotomy gap also reveals fluid collections which may require surgical intervention (Fig. 4).

Small cube-shaped dressings at the site of wire entry to the skin are treated daily with 1-2 cc povidone-iodine to prevent infection. The dried accumulation of iodine is dense enough to be radiographically evident on plain films. Care must be taken to ensure they are not mistaken for new bone formation in the corticotomy site when they project over this region.

\section{Discussion}

The standard techniques for limb lengthening are difficult orthopedic procedures with high complication rates $[5,9]$. The newly available Ilizarov technique has been utilized 


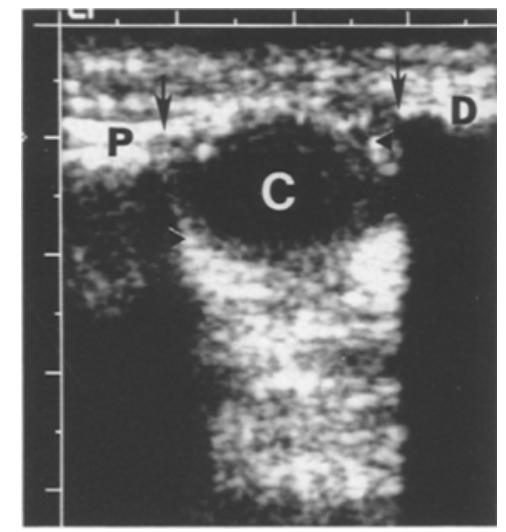

Fig.4. The longitudinal sonogram of the tibia $(\mathrm{P}=$ proximal, $\mathrm{D}=$ distal) reveals the gap (arrows) and new linear streaks of bone (arrowheads) and a fluid collection (C) in the corticotomy gap

in the Soviet Union since the early 1960's and in Western Europe since 1981. The incidence of serious complications has been reported to be significantly lower than other current techniques $[1,5,9]$. The Ilizarov method induces regenerate bone formation under controlled progressive tension-stress. This has been termed distraction histogenesis [5] or callotasis [10]. This technique is unique in the method used to induce osteogenesis. Aronson and colleagues, using a canine model to reproduce Ilizarov's model, evaluated the regenerate bone, and found several factors in this technique to be important: the latency period, the rate of distraction, and the rigidity of the transfixing wires $[11,12]$.

Problems with imaging the Ilizarov lengthened limbs can be avoided. It is important that the plain films be centered accurately to the distraction site and the connecting posts aligned outside the distraction gap. This involves making the technologists aware of the purpose of the films. In evaluating the distraction gap for the presence of new bone formation, the iodine soaked dressings should not be mistaken for regenerate bone formation. In providing accurate measurement of the distraction gap some correction must be made for the magnification since the apparatus lifts the limb away from the film. We have found that a ruler attached with a Velcro band along the side of the limb at the level of the gap provides an easy reference.

Plain fim radiographic appearance of new bone formation can be delayed [6]. Sonography provides earlier information on new bone formation in addition to accurate measurements of the distraction gap. In children, rate of bone formation is accelerated compared to adults. Overly fast distraction inhibits bone formation while overly slow distraction can lead to premature consolidation prior to achieving new length. Sonography can play an important role in documenting new bone formation prior to its radiographic appearance and so guide the orthopedic surgeon on appropriate distraction rates to maintain good bridging of the regenerate bone in this new limb lengthening procedure [6-8]. In addition, ultrasound can be used to accurately measure the distraction gap without ionizing radiation and document fluid collections that may require surgical intervention $[6,7]$. Thus a combination of alternating plain radiographs and ultrasound could be utilized to follow the progress in these children.

\section{References}

1. Ilizarov GA (1973) Operative elongation of the femur. Ortop Travmatol Protez 34: 51

2. Ilizarov GA (1989) The tension stress effect on the genesis and growth of tissues: Part I. The influence of stability of fixation and soft-tissue preservation. Clin Orthop 238: 249

3. Ilizarov GA (1989) The tension stress effect on the genesis and growth of tissues: Part II. The influence of the rate and frequency of distraction. Clin Orthop 239:263

4. Ilizarov GA (1990) Clinical application of the tension-stress effect for limb lengthening. Clin Orthop 250: 8

5. Paley D (1988) Current techniques of limb lengthening. J Pediatr Orthop 8: 73

6. Young JWR, Kostrubiak IS, Resnik CS, Paley D (1990) Sonographic evaluation of bone production at the distraction site in Ilizarov limb-lenghtening procedures. Am J Roentgenol 154: 125

7. Huppertz R, Pfeil J, Kaps HP (1990) Sonographische Verlaufskontrollen von Verlängerungsosteotomien. Z Orthop 128: 90

8. Galante VN, Angelelli G, Caiaffa V, Macarini L (1989) Il controllo ecografico nei pazienti sottoposti ad allungamento degli arti secondo la metodica di Ilizarov. Minerva Ortop Traumatol 40: 529

9. Vade A, Eissenstat R (1990) Radiographic features of bone lengthening procedures. Radiology 174:531

10. De Bastiani G, Aldegheri R, Renzi-Brivio L, Trivella G (1987) Limb lengthening by callus distraction (callotasis). J Pediatr Orthop $7: 129$

11. Aronson J, Harrison B, Boyd CM, Cannon DJ, Lubansky HJ (1988) Mechanical induction of osteogenesis: the importance of pin rigidity. J Pediatr Orthop 8: 396

12. Aronson J, Good B, Stewart C, Harrison B, Harp J (1990) Mechanical induction of osteogenesis: the importance of pin rigidity. Clin Orthop 250: 43

C.E.Blane, M.D.

Section of Pediatric Radiology

C. S. Mott Children's Hospital C3123/0252

1500 E. Medical Center Drive

Ann Arbor, MI 48109-0252

USA 